\title{
La contaminación proveniente de la industria curtiembre, una aproximación a la realidad ecuatoriana
}

Fecha de recepción: 2021-09-24 • Fecha de aceptación: 2021-11-23 • Fecha de publicación: 2022-01-10

\author{
María José Silva ${ }^{1}$ \\ Universidad de Palermo, Argentina \\ mariajose_3019@yahoo.com \\ https://orcid.org/0000-0003-1464-5937
}

Diego Salinas Morales ${ }^{2}$

BH Consultores, Ecuador

dsalinas@bhconsultores.com

https://orcid.org/0000-0001-8261-648X

\section{Resumen}

El presente artículo se centró en describir los procesos típicos, materiales utilizados, principales riesgos para la salud derivados de la industria del curtido de pieles, así como el impacto ambiental asociado a dicha industria y actividad en la provincia de Tungurahua, Ecuador. Para el desarrollo del presente documento se realizó una revisión integradora de la literatura, como una modalidad de investigación bibliográfica. Como resultado principal se obtuvo que la contaminación de los afluentes hídricos, con químicos provenientes de las aguas residuales, producto de la transformación de las pieles animales en cuero para la industria de la marroquinería, ha determinado el crecimiento exponencial de la contaminación, debido a la deficiente gestión que se otorga a dichos residuos. La contaminación de los recursos hídricos se evidencia en la cantidad de cromo vertido en el agua, tanto de tipo trivalente, como hexavalente. La concentración de Cr6 va desde los 52.3 a los 392.9 $\mathrm{mg} / \mathrm{L}$, rebasando el límite que determina la normativa TULAS. Se concluye que la determinación de $\mathrm{Cr} 6$ se convierte en un potencial factor y un riesgo inminente para el desarrollo de las diferentes afectaciones asociadas a la exposición al cromo. 
Palabras clave: curtiembre, curtidurías, contaminación, cromo, gestión ambiental, pieles animales

\begin{abstract}
This article focused on describing the typical processes, materials used, main health risks derived from the leather tanning industry, as well as the environmental impact associated with this industry and activity in the province of Tungurahua, Ecuador. For the development of this document, an integrative review of the literature was carried out, as a bibliographic research modality. The main result was that the contamination of water tributaries with chemicals from wastewater, resulting from the transformation of animal skins into leather for the leather goods industry, has determined the exponential growth of contamination, due to the poor management of such waste. Contamination of water resources is evidenced by the amount of chromium discharged into the water, both trivalent and hexavalent. The concentration of $\mathrm{Cr} 6$ ranges from 52.3 to $392.9 \mathrm{mg} / \mathrm{L}$, exceeding the limit determined by the TULAS regulations. It is concluded that the determination of Cr6 becomes a potential factor and an imminent risk for the development of different affectations associated with chromium exposure.
\end{abstract}

Keywords: tannery, tanneries, contamination, chromium, environmental management, animal skins 


\section{Introducción}

La industria curtiembre trata del procesamiento de pieles, usualmente de ganado, lo cual es tratado para covertir en cuero, material que es utilizado en calzado, tapiceria, vestimenta, entre otros. La crianza del ganado, sea ovino o bovino, la alimentación y cercos de crianza, tienen relacion directa con la calidad de la piel, además, son aspectos importantes para el tratamiento de este material. Es decir, la forma de vida de los animales que son parte de esta industria es relevante, debido a que a partir de esto, el procesamiento de la piel será más o menos fuerte, según la circunstancia. Por lo cual, si la piel es muy maltratada, esto conlleva a mayor uso de químicos y manipulaciones por parte de los trabajadores. Apartir de esto es que puede ser utilizado en la fabricación de otros productos (Organización de las Naciones Unidas para la Agricultura y la Alimentación, 2010).

Esta es una industria de alta exigencia a nivel mundial, que se a desarrollado más en Europa y Estados Unidos a partir del siglo XX, debido a la fuerte industrialización del material, ya que es exigido por muchas áreas comerciales, en su mayoría, ligados a la moda. Esto a incentivado al uso de nuevas herramientas tecnológicas, químicos y explotar el mercado. En consecuencia, Latinomerica ha tenido un incremento a solventar, en razón de que en muchas zonas no se encuentra este tipo de equipos que permitan explotar esta producción curtiembre. A pesar de eso, los latinoamericanos se han mantenido en este mercado, sin dejar de lado los requerimientos de los consumidores, la industria, asociaciones gremiales y sindicales que ponen lineamientos para su libre venta (Méndez et al., 2007).

Por otro lado, desde el tener un producto, hasta ponerlo a la venta, lleva un proceso, en esto, como primera estancia, se tiene que comprar la piel en bruto, que usualemente es recién sacada de los animales, a veces, al adquirir este producto residual se tiene restricciones por cuestión de ser un subproducto de la carne, por lo cual se necesitan cumplir con los lineamientos de salubridad, de esta manera se asegura que el producto no es un material que pueda afectar la salud de las personas. Seguido a esto viene el proceso de curtición, que es por medio de varios procesos químicos y lavados, para de esta forma poder realizar los acabados de este material que tiene que ver con la textura, color y calidad del cuero, seguro de la fabricación de productos como calzado, tapicería, confección, entre otros. Finalmente, tener como resultado la venta de los artículos en cualquiera de sus presentaciones (Núñez \& Orozco, 2016).

En el Ecuador, de forma resumida, el proceso de curtiembre trata de sacar el pelo de la piel, curtir con agentes de curtimiento que usualmente son químicos, de esta forma dar características físicas como textura, color y engrasado al material para un mejor aspecto y tacto, según en lo que se utilice. Es una industria de poca explotación y conocimiento en el país, lo cual se puede deber a que no hay la tegnologia suficiente para su explotación; sin embargo, la provincia de Tungurahua es la de mayor producción de este tipo. Dentro del país hay aproximadamente, entre empresas artesanales grandes, mediana y pequeñas, un total de 80 curtiembres, además de 60 empresas y personal que expende los productos para el tratamiento del cuero. Esto significa que es una fuente productiva del país, además de ser una plaza de trabajo en diferentes etapas en el tratamiento del cuero (Cerón, 2011). 
Posterior a la producción de cuero quedan los subproducto o productos residuales, como por ejemplo, productos solidos del pelo, restos de carnaza, cal, sulfuros, grasas, restos de cuero, polvo de curtido, aerosoles solidos, entre otros. Este es uno de los aspectos que más dificultad trae la industria curtiembre, la contaminación. Es una parte de poca atención, lo cual requiere reformas ambientales a nivel industrial en este sector. Ante esta demanda, Ecuador, con las reformas ambientales para la curtiembre, busca que la producción de este material sea sustentable ambientalmente, y por ende, bajar los niveles que afectan no solo al país, si no al mundo en general, haciendo esta actividad más amigable con el ecosistema (Cando, 2012; Ministerio de Ambiente y Agua, 2011b).

Además de los residuos solidos, se encuentran los residuos de liquidos y químicos, como lo son los minerales, este tipo de elementos pueden ser de alto riesgo en la salud, tanto en las personas externas, como para quienes trabajan con este tipo de productos. Entre los elementos que salen de la curtiembre se tiene el cloruro de sodio, demanda biológica de oxigeno (DBO), demanda química de oxigeno (DQO), sulfuros (S), amonio, nitrógeno orgánico, entre otros. Respecto a sustancias peligrosas se identifica arilaminas, pentaclorofenol (PCP), formaldehido, tributilestaño (TBT), mercurio, cadmio, zirconio y cromo. Este último es el reto más grande en el tratamiento de los afluentes y se encuentra relacionado con el cáncer, afecciones de la piel e intoxicaciones. Debido a esto, algunos lugares como Lánus y Avellaneda en Buenos Aires, procuran reciclar los licores de cromo y reutilizarlos (Vásquez \& Gonzales, 2009).

Los ríos Ambato y Pachanlica, principales fuentes hídricas de la región, presentan un alto contenido de cromo, plomo, hierro, grasas, plaguicidas, bacterias coliformes fecales y otros derivados procedentes de la curtiembre, así como de otras industrias relacionadas con la fabricación de alimentos, ropa y papel, al verter sus desechos, incrementando el riesgo de afectaciones al agro, los animales de la zona y salud de la población de la región (Medina, 2013). Del total de la actividad curtiembre de Ecuador, se estima que aproximadamente el $80 \%$ de la industria efectúa sus actividades dentro de la provincia de Tungurahua, y al ser dicha actividad económica un gran motor laboral, de la misma forma, tal actividad se convierte en un gran foco generador de contaminación ambiental (Masabanda et al., 2017).

La gestión ambiental, al no conformar un elemento de prioridad en la administración de la industria curtiembre y, agregando el desconocimiento de la normativa legal vigente en materia de gestión residual, la falta de tecnología, y el deficiente presupuesto para el adecuado tratamiento de los mismos, son factores prominentes de la intencional contaminación de la industria hacia las fuentes acuíferas (González et al., 2017). Esto llama la atención de las entidades legislativas, con el objetivo de tener seguimiento de este fenómeno para tener mayor apoyo y poner lineamientos más rigidos, además de capacitar a la ciudadanía sobre la forma adecuada de tratamiento residual.

Son todas estas situaciones, tanto las actividades curtiembres, la falta de gestión ambiental, y la poca atención por parte de las instituciones gubernamentales, que han hecho que los ríos de Ambato y Pachanlica tengan un nivel de contaminación elevado (Galarza \& Santana, 2016). A continuación, el objetivo del presente estudio es describir los procesos típicos, materiales utilizados, principales riesgos para la salud derivados de la industria del curtido de pieles, así como el impacto ambiental asociado a dicha industria y actividad en la provincia de Tungurahua. 


\subsection{Transformación de las pieles animales}

Respecto a la transformacion de las pieles de animales, en la Enciclopedia de Salud y Seguridad en el Trabajo de la Organización Internacional del Trabajo (OIT), es definida como un "proceso químico mediante el cual se convierten los pellejos de animales en cuero" (Mccann, 2001). Lo cual consiste en el empleo de álcalis, ácidos, enzimas, sales y agentes curtientes, con la finalidad de reforzar la estructura proteica del cuero animal. De esta forma, hacerlo más firme y grueso para el empleo en la fabricación de carteras, zapatos, correas, guantes, prendas de vestir y derivados, que usualmente se comercializan nacional e internacionalmente.

En el Ecuador, en los camales o lugares de producción gandera es donde se adquieren las pieles que se utilizan en la industria curtiembre, estas provienen principalmente del ganado bovino, porcino y ovino como subproducto de la manufactura cárnica. Es decir, que se utilza el subproducto proveniente de los productos cárnicos, los cuales de no ser utilizados, las pieles deberían desecharse o incinerarse para evitar mayor contaminación (Ministerio de Ambiente y Agua, 2011a).

Tabla 1.

Procedimiento para el tratamiento de pieles

\begin{tabular}{ll}
\hline 1 & Recepción de la materia prima \\
\hline 2 & Pre-tratamiento (clasificación de las pieles por tipo) \\
\hline 4 & Curado y desinfectado (Salazón húmeda o curado con sal muera) \\
\hline 5 & Pelambre (eliminación de la epidermis y pelo) \\
\hline 6 & Descarnado (separación de grasas y carnazas) \\
\hline 7 & Desengrasado (Eliminación de proteínas) \\
\hline 8 & Piquelado (preparación de la piel para el curtido con ácidos) \\
\hline 9 & Curtido (con cromo o agentes vegetales) \\
\hline 10 & Secado (secar el cuero para determinar sus propiedades finales) \\
\hline 11 & Engrasado (engrasar el cuero para determinar sus texturas) \\
\hline 12 & Planchado y clasificación (brindan satín o brillo al cuero) \\
\hline
\end{tabular}

Fuente: Ministerio de Ambiente y Agua (2011a)

Para el proceso del curtido de pieles se puede emplear dos tipos de procedimientos convencionales, el primero en base a sales de cromo, siendo el método que el $80 \%$ de la industria lo utiliza, también determinado como el más contaminante y, como segunda opción, el proceso que implica la utilización de agentes vegetales que cumplen la misma función que el primero, pero con menor eficacia, con más empleo de tiempo que el anterior, y más amigable con el medio ambiente (Chávez, 2010; Ministerio de Ambiente y Agua, 2011a). En la Tabla 1 se presenta el proceso de trataminto del cuero independientemente del proceso que se emplee para el curtido de pieles, 
tanto con agentes vegetales, como con sales de cromo, el procedimiento cumple con una serie de pasos preestablecidos para obtener el producto final.

\section{Metodología}

Se efectuó una revisión integradora de la literatura, centrándose en revisar y sintetizar datos secundarios sobre el tema de investigación de manera integrada, de modo que se generan nuevas perspectivas sobre el tema. Se utilizó la navegación en Internet de la base de datos de Google Scholar, Scielo y Sciencedirect.com para identificar y descargar resúmenes y artículos de investigación relacionados con la contaminación, proveniente de la industria curtiembre, se utilizaron parámetros de búsqueda como: Curtiduría + Riesgos + Tratamientos + Físico + Biológico + Químico. La base para la selección de artículos se centró en procedimientos generales en el tratamiento del cuero, descripción de procedimientos, equipos y métodos adoptados.

\section{Resultados}

\subsection{Aguas residuales como resultado del proceso de curtiembre}

Según Greenpeace (2012), para obtener una tonelada de cuero terminado se estima que son necesarios aproximadamente $500 \mathrm{~kg}$ de agentes químicos para su transformación, de los cuales el $85 \%$ de dichos productos se convierten en desechos residuales del proceso, que al final son elementos tóxicos para cualquier ser vivo. Sin olvidar como desecho también a los restos de animales como piel cruda, grasa y pelo, que se descarta que al combinarse para su eliminación, produce un considerable volumen de residuos líquidos y sólidos que son vertidos a los afluentes hídricos de las regiones, determinando así el alto nivel de contaminación de la industria curtiembre. Al ser esto expedido en los ríos, se provoca una contaminación en fauna, eliminando a los seres como peses, larvas naturales del agua y flora como plantas acuáticas. Es decir, que se eliminan los agentes que dan vida natural, lo cual tiene un impacto en todo el ecosistema, colaborando incluso con el calentamiento global (Soto, 2015).

Después del proceso de curtido en las aguas residuales resultantes, el agente químico con mayor presencia es el Cromo $(\mathrm{Cr})$ en su forma trivalente $\left(\mathrm{Cr}^{3}\right)$, que es menos tóxica, pero dicho elemento puede transformarse a su forma hexavalente $\left(\mathrm{Cr}^{6}\right)$, en dependencia de las características propias del agua que ayuden a la oxidación del $\mathrm{Cr}^{3}$ (Medina, 2013). El $\mathrm{Cr}^{6}$ es conocido como un elemento más inestable y 1.000 veces más tóxico que el $\mathrm{Cr}^{3}$ (Chávez, 2010). Las aguas residuales, producto del proceso de curtiembre por el método convencional de sales de cromo, son una de las fuentes principales de contaminación ambiental por cromo en vertientes de agua dulce (Borda-Prada, 2014).

Además, el uso indiscriminado e ineficiente de agua para el procesamiento del cuero, 1000 litros de agua por cada cuero procesado aproximadamente, crean un volumen considerable de desechos líquidos y sólidos que se vierten constantemente a los afluentes (Greenpeace, 2012), por esa razón, es de vital importancia por parte de los organismos ambientales rectores, el control 
exhaustivo y tratamiento de dichas descargas (Medina, 2013). De esta forma se previene que estas negligencia causen afecciones graves en la salud de todos los seres vivos

\subsection{Riesgos y salud}

Para Amalberti (2009), en su apartado denominado la exposición inconfesable a los riesgos, menciona que la exposición a los riesgos asociados al trabajo continúa siendo un tema poco reconocido por la seguridad del trabajo. Sin embargo, recomienda diferenciar las exposiciones a los diferentes tipos de riesgo: (1) las exposiciones voluntarias de las (2) exposiciones involuntarias, determinando así a las exposiciones involuntarias como la exposición del individuo a riesgos del trabajo no imaginados, no deseados o no aceptados, hasta la manifestación oficial de los riesgos y consecuencias asociadas.

Dentro de esta categoría se han tipificado también todo tipo de prácticas laborales mal intencionadas. Los principales riesgos de la industria curtiembre, según Mccann (2001), se asocian con: riesgos de infección, accidentes, riesgo mecánico, riesgo ergonómico, polvo, pero por sobre todos estos, se encuentra el riesgo químico, debido al empleo de una extensa cantidad de taninos, álcalis, ácidos, desinfectantes y disolventes, que son irritantes y causan afectaciones a la piel y las vías respiratorias, produciendo dermatitis por contacto y bronquitis, respectivamente como mínimo impacto a la salud.

Para el Ministerio de Sanidad Consumo y Bienestar Social (2001), en su apartado sobre los perjuicios a la salud ligados al trabajo, en su numeral dos, determinan a las patologías ligadas al ambiente como "las enfermedades infecciosas, las alergias, las intoxicaciones relacionadas con la presencia, en la situación de trabajo, de agentes biológicos y de productos químicos. Los cánceres profesionales pertenecen a esta categoría" (p. 54). Esto quiere decir que en ciertas ocasiones, como es el caso de los residuos de la producción cuertiembre, la exposición, tanto de los trabajadores, como de cualquier persona, puede afectar la salud en diferentes niveles, ya sea leves o graves, por ejemplo, la adquisición de una enfermedad maligna como el cáncer.

En el caso de las curtiembres y sus procesos, distintos organismos internacionales encargados de la salud y su prevención como la Agencia Internacional para la Investigación del Cáncer (IARC) y el Departamento de Salud y Servicio Humanos (DHSS) con sede en Estados Unidos, determinan al Cromo Hexavalente $\left(\mathrm{Cr}^{6}\right)$ como un elemento cancerígeno (Medina, 2013), así como un elemento de complicada biodegradación, debido a su persistencia en el ambiente y sus efectos inesperados en la vida acuífera (Greenpeace, 2012).

Debido a la exposición intensa y prolongada, la inhalación de químicos con contenido de cromo en trabajadores de esta industria puede generar enfermedades como el cáncer sinonasal, cáncer de páncreas y cáncer de pulmón, así como cáncer de vejiga y también cáncer testicular (Mccann, 2001). De igual forma, la ingesta de $\mathrm{Cr}^{6}$ en humanos muestra una sintomatología gastrointestinal con presencia de vómitos, hemorragias y diarrea que puede derivar una insuficiencia renal aguda, y posteriormente la muerte por un colapso cardiocirculatorio (Chávez, 2010). Los efectos del $\mathrm{Cr}^{6}$ en animales, que asimilan este elemento por vía oral, son úlceras e inflamaciones estomacales, a 
nivel de intestinos, así como afectaciones a nivel reproductivo, especialmente en el macho de la especie por las alteraciones en su esperma (Medina, 2013).

\subsection{Gestión de residuos}

Respecto a la gestión de residuos, la totalidad de las industrias de la curtiduría, con el fin de mantenerse y ser sostenible, están obligadas al cambio de sus procesos y prácticas industriales, desafiando a la tecnología de forma directa, aunque dicho panorama se torne difícil e incluso inaccesible en ciertas circunstancias (Guerrero, 2014)Calle 163 No.54 C 34, 111/3. RESUMEN Se presenta en este artículo un análisis de la sostenibilidad individual y sectorial de pequeños y medianos negocios dedicados a la transformación de la piel animal en el municipio de Villapinzón (Colombia. Es de suma importancia que el sector curtiembre invierta en nuevas tecnologías, con la finalidad de mejorar sus vertidos contaminantes, así como la implementación de la extracción de los metales del agua para su debida reutilización o eliminación (Masabanda et al., 2017).

Al tener conocimiento de esto, la verdad de la inexistente gestión ambiental en la industria radica en el desinterés, la alta informalidad, el mercado no exigente, pero sobre todo, en la incapacidad de las autoridades ambientales que no ejercen ni exigen acatar el respectivo control de la normativa ambiental vigente (González et al., 2017). Para el cumplimiento de dicha normativa, es fundamental que los organismos rectores desplieguen un estricto control, así como un sistema moderador que garantice la aplicación de todas las disposiciones, a fin de generar conciencia a propietarios y trabajadores de la industria, sobre los daños inminentes al ecosistema, pero sobre todo, a la salud de las personas de la región (Galarza \& Santana, 2016)

Varios son los métodos que se pueden emplear para el tratamiento de las aguas residuales producidas por el proceso de la curtiduría de pieles, y queda a criterio personal y de la industria cuál método tendría mejor eficacia para su gestión residual. Entre los principales procesos se encuentran la precipitación de cromo con hidróxido de sodio, usando poliacrilamidas como floculante; la fitorremediación; la remoción de cromo, utilizando mallas moleculares; la absorción de cromo sobre carbones activados modificados; el uso de hidróxido de calcio (o cal apagada: $\mathrm{Ca}[\mathrm{OH}] 2$ ), hidróxido de sodio y carbonato de sodio en el tratamiento de efluentes del proceso de curtido al cromo, y el uso de residuos agrícolas para reducir cromo hexavalente $(+6)$ a cromo trivalente (+3) (Borda-Prada, 2014, p.7)

Esto como formas de tratamiendo de curtiembre que se realizan con el objetivo de tener como producto final el cuerpo listo para su empleo, en diferentes artículos de consumo.

Además de lo antes mencionado, existen procesos de tratamiento de los productos de la cuertiembre que son de uso tanto en en las empresas grande, como por las pequeñas. En esto se conoce que existen tratamientos primarios y secundarios, dentro del tratamiento primario constituyen operaciones unitarias de sedimentación primaria, tamizado, coagulación-floculación, flotación y sedimentación secundaria. Este tipo de tratamiento es necesario para aquellos procesos de pelambre y de curtido, debido a la alta presencia de carga contaminante de materia orgánica, sólidos suspendidos, sulfuros, y cromo, entre otros (Masabanda et al., 2017, p.58) 
A pesar de ser métodos contaminenates que pueden afectar la salud y el ecosistema en general, es importante realizarlo, ya que gracias a eso es que se puede hacer factible el uso del cuero en la industria.

\section{Conclusiones}

Ante los contaminantes, encontramos las concentraciones de $\mathrm{Cr}^{6}$ en el agua, después del vertido de desechos determinados por el estudio -determinación de cromo hexavalente en descargas de aguas residuales de una curtiembre mediante espectrofotometría de absorción atómica-, del presente trabajo que determina que la concentración de $\mathrm{Cr}^{6}$ va desde los 52.3 a los $392.9 \mathrm{mg} / \mathrm{L}$, rebasando el límite que determina la normativa TULAS, por consiguiente, dicha determinación se convierte en un potencial factor y un riesgo inminente para el desarrollo de las diferentes afectaciones asociadas a la exposición al cromo detalladas anteriormente.

La contaminación de los recursos hídricos de la provincia de Tungurahua se evidencia en la cantidad de cromo vertido en el agua, tanto de cromo trivalente, como de cromo hexavalente, así como de otros desechos sólidos como consecuencia del proceso ineficiente en el tratamiento de desecho de pieles. La cantidad de cromo hexavalente permisible en el agua, según el Ministerio de Ambiente y Agua (2011b), en su publicación Texto unificado de legislación ambiental secundario (TULAS), Norma de Calidad Ambiental y de descarga de efluentes, es de $0.5 \mathrm{mg} / \mathrm{L}$.

Es trascendental que la industria de la curtiría se plantee la implementación de medidas correctivas que contengan una producción más eficiente y amigable con el ambiente, sin olvidar el cambio de tecnologías, la normativa ambiental vigente, pero sobre todo, la prevención de los riesgos asociados al proceso curtiembre con cromo en la salud de los trabajadores y de la población. 


\section{Referencias}

Amalberti, R. (2009). Acción humana en los sistemas sistemas de alto riesgo. Modus Laborandi.

Borda-Prada, O. (2014). Evaluación y reducción de los niveles de cromo en muestras de aguas residuales provenientes de curtiembres. Revista L'estrit Ingénieux, 5(1), 6-12. http://revistas.ustatunja.edu.co/index. php/lingenieux/article/view/1230

Cando, D. (2012). Recurtimiento de pieles caprinas con la utilización de diferente niveles de recurtiembre vegetal guarango. [Tesis de grado, Escuela Superior Politécnica de Chimborazo]. DSpace ESPOCH. http:// dspace.espoch.edu.ec/handle/123456789/1856

Cerón, P. (2011). Estudio de un sistema físico-químico a escala prototipo de tratamiento de aguas residuales provenientes de una curtiembre. [Tesis de grado, Universidad San Francisco de Quito Colegio]. Repositorio Digital USFQ. http://repositorio.usfq.edu.ec/handle/23000/1248

Chávez, Á. (2010). Descripción de La nocividad del cromo proveniente de la industria curtiembre y de las posibles formas de removerlo. Revista Ingenierías Universidad de Medellín, 9(17), 41-49. http://www.scielo. org.co/pdf/rium/v9n17/v9n17a04.pdf

Galarza, J., \& Santana, A. (2016). Normas ambientales y competitividad en las pymes del sector curtiembre de Ambato-Ecuador. Ojeando la Agenda, (44), 54-74. https://mirevistadigital.files.wordpress.com/2016/11/ art-cumplimiento-de-las-normas.pdf

González, A., Alaña, T., \& Gonzaga, S. (2017). La gestión ambiental en la competitividad de las PYMES del Ecuador. INNOVA Research Journal, 2(8.1), 236-248. https://doi.org/10.33890/innova.v2.n8.1.2017.371

Greenpeace. (14 de marzo de 2012). Nuevas evidencias de contaminacion en la Cuenca Mataza-Riachuelo. https://www.greenpeace.org/archive-argentina/es/informes/Cueros-toxicos/

Guerrero, M. (2014). Sostenibilidad de pequeñas y medianas curtiembres de Villapinzón. In Congreso Internacional de Logística y SCM. Memorias (pp. 169-178). DOI:10.13140/2.1.3075.5520

Masabanda, M., Echegaray, C., y Bartolomé, A. (2017). Control de la contaminación en aguas residuales de curtiembres, mediante fotocatálisis heterogénea con TiO2. Revista de Ciencias de Seguridad y Defensa, 2(1), 57-77. http://geo1.espe.edu.ec/wp-content/uploads/2017/10/art6.pdf

Mccann, M. (2001). Cuero, pieles y calzado. In Industrias Textiles y de confección (pp. 88.1-88.13). Ministerio de Trabajo y Asuntos Sociales. https://www.insst.es/documents/94886/161971/Capítulo+88.+Cuero,+pieles+y+calzado 
Medina, M. (2010). Determinación de cromo hexavalente en descargas de agua residuales de una cuetiembre, ubicada en el sector de Izamba, Ambato ne la provincia de Tungurahua, mediante espectrofotometría de absorción atómica. [Tesis de grado, Pontificia Universidad Católica del Ecuador. Repositorio PUCE. http:// repositorio.puce.edu.ec/bitstream/handle/22000/3162/T-PUCE-2785.pdf? sequence=1\&isAllowed=y

Méndez, R., Vidal, G., Lorber, K., \& Márquez, F. (2007). Producción limpia en la industria de curtiembre. Universidade de Santiago de Compostela. http://www.eula.cl/giba/wp-content/uploads/2017/09/produccion-limpia-en-la-industria-de-curtiembre.pdf

Ministerio de Ambiente y Agua. (2011a). La industria de los cueros (a base de sales de cromo, con agentes vegetales). In Estudio para conocer los potenciales impactos ambientales y vulnerabilidad relacionada con las sustancias químicas y tratamiento de desechos peligrosos en el sector productivo del Ecuador (pp. 127-247). Ministerio de Ambiente y Agua. https://www.ambiente.gob.ec/wp-content/uploads/downloads/2013/03/PART2.pdf

Ministerio de Ambiente y Agua. (2011b). Revisión y actualización de la norma de calidad ambiental y de descarga de efluentes: recursos agua. Ministerio de Ambiente y Agua.

Ministerio de Sanidad Consumo y Bienestar Social. (2001). Protocolo de vigilancia sanitaria específica para los/ as trabajadores/as expuestos a agentes biológicos. Ministerio de Sanidad y Consumo. https://www.mscbs. gob.es/ciudadanos/saludAmbLaboral/docs/agentes biologicos.pdf

Núñez, M., \& Orozco, J. (2016). Implementación de un bombo de acabados del cuero para el estudio del proceso de recurtido y tinte de cueros de especies menores para el laboratoro de ingeniería agroindustrial de la Universidad Nacional de Chimborazo. [Tesis de grado, Universidad Nacionl de Chimborazo]. Universidad Nacional de Chimborazo. http://dspace.unach.edu.ec/handle/51000/1876

Organización de las Naciones Unidas para la Agricultura y la Alimentación. (2010). Perspectivas a plazo medio de los productos básicos agrícolas. http://www.fao.org/3/y5143s/y5143s.pdf

Soto, E. (2015). El calentamiento global y la degradación de la ozonosfera (Un análisis científico desde la diversidad de criterios). Revista Integra Educativa, 8(3), 43-54. http://www.scielo.org.bo/pdf/rieiii/v8n3/ v8n3 04.pdf

Vásquez, J., \& Gonzales, D. (2009). Metodología para implementar un mdelo de responsabilidades sociales empresarial (RSE) en la industria de la curtiembre en Colombia. Contabilidad y Negocios, 4(8), 49-56. http://revistas.pucp.edu.pe/index.php/contabilidadyNegocios/article/view/937/904 
Copyright (c) 2022 María José Silva y Diego Salinas Morales

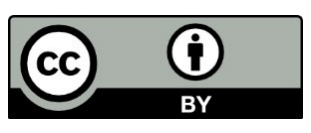

Este texto está protegido bajo una licencia internacional Creative Commons 4.0.

Usted es libre para Compartir-copiar y redistribuir el material en cualquier medio o formato - y Adaptar el documento - remezclar, transformar y crear a partir del material-para cualquier propósito, incluso para fines comerciales, siempre que cumpla las condiciones de Atribución. Usted debe dar crédito a la obra original de manera adecuada, proporcionar un enlace a la licencia, e indicar si se han realizado cambios. Puede hacerlo en cualquier forma razonable, pero no de forma tal que sugiera que tiene el apoyo del licenciante o lo recibe por el uso que hace de la obra.

\section{Resumen de licencia - Texto completo de la licencia}

